\title{
The Universal Cross-Section of Photonic Interaction
}

\author{
Richard A. Hutchin \\ Optical Physics Company, Calabasas, USA \\ Email: rahutchin@opci.com
}

Received 18 January 2015; accepted 12 May 2015; published 15 May 2015

Copyright (C) 2015 by author and Scientific Research Publishing Inc.

This work is licensed under the Creative Commons Attribution International License (CC BY). http://creativecommons.org/licenses/by/4.0/

c) (i) Open Access

\begin{abstract}
In 1917, Einstein published his paper [1] renowned for the discovery of stimulated emission. However, it also contained the principles which allowed the calculation of the universal photonic cross-section $=\lambda^{2} / 2 \pi$. Any heavy electronic system will have this cross-section for photonic excitation and stimulated emission in its rest frame. The modifications of this cross-section due to the recoil of the emitter have not yet been calculated, but are in general expected to be second order in recoil velocity.
\end{abstract}

\section{Keywords}

Photonic Cross-Section, Stimulated Emission, Excitation

\section{Introduction}

When Einstein first found the term for stimulated emission, he based that discovery on some very simple physics, including the principle that the radiant spectrum of blackbody equilibrium was universal. We still believe all these principles today. Using those same results and some algebra, we find that they also imply a universal cross-section for every type of photonic interaction, from nuclear to atomic to superconducting.

\section{Short Overview of Einstein's Calculation}

A very brief summary is presented here to highlight Einstein's logic, which we will then extend. He began with a two state electronic system in thermal equilibrium at temperature $T$, which produced a probability $P_{1}$ of being in the ground state and probability $P_{2}=1-P_{1}$ of being in the excited state. For simplicity, he assumed that the excitable system had identical excitation and emission wavelengths, which meant that it had no recoil during emission or excitation (absorption) and was thus infinitely massive. This was a simplifying assumption and will also apply to the derivation which follows. 
He also applied the known physics for the blackbody spectrum $B B(v)$, where $v=$ the frequency of light, and the known equilibrium thermal probability ratio $P_{2} / P_{1}$ for the two states as summarized below, where the additional physics of $E=h v$ was also known and used.

$$
\begin{aligned}
& B B(v)=\frac{8 \pi h h}{\mathrm{e}^{\frac{h v h h}{k T}}-1} \frac{v^{3}}{c^{2}} \\
& \frac{P_{2}}{P_{1}}=\mathrm{e}^{\frac{-E_{12}}{k T}}=\mathrm{e}^{\frac{-h v}{k T}}
\end{aligned}
$$

Then he assumed three processes that would change the electronic state back and forth: spontaneous emission, excitation and finally stimulated emission. Stimulated emission was unknown to physics at that time but was required in order to achieve the proper equilibrium given by the mean rate of excitation minus de-excitation being zero. Since he assumed everything would be in thermal equilibrium, the rate of change of the probability of being excited must be zero on average. This was his key physics principle shown in Equation (3). Here the three constants $A_{21}$ (spontaneous emission), $B_{21}$ (stimulated emission) and $B_{12}$ (excitation) can all be adjusted to make this equation work. For later reference we also point out that constant $A_{12}=1 / \tau_{\text {decay }}$, where $\tau_{\text {decay }}$ is the exponential decay time of state 2 into state 1 . This is the known decay form for a state at rest without other disturbances as described by quantum mechanics, and was also well known in Einstein's time. This type of exponential decay produces a familiar line shape, called a Lorentz line shape, which we will use later.

$$
\frac{\mathrm{d} P_{2}}{\mathrm{~d} t}=-A_{21} P_{2}-B_{21} P_{2} B B(v)+B_{12} P_{1} B B(v)=0
$$

Dividing Equation (3) by $P_{1}$ and substituting from Equations (1) and (2), we get Equation (4).

$$
-A_{21} \mathrm{e}^{-\frac{h v}{k T}}+\left(B_{12}-\mathrm{e}^{-\frac{h v}{k T}} B_{21}\right)\left(\frac{8 \pi h}{\mathrm{e}^{\frac{h v}{k T}}-1} \frac{v^{3}}{c^{2}}\right)=0
$$

which is identically zero if and only if the following two conditions apply, as summarized in Equations (5a) and (5b).

$$
\begin{aligned}
A_{21} & =\frac{B_{21} 8 \pi h v^{3}}{c^{2}} \\
B_{12} & =B_{21} .
\end{aligned}
$$

\section{Computing the Universal Photonic Cross-Section}

Back in 1917 the main attention focused on the $B_{21}$ term for stimulated emission, which was new to physics. Here however, we wish to focus on the first two terms which will allow us to compute the universal cross-section of a photonic interaction. Our process will be to write the decay rate in two different, physically equivalent forms:

$$
\frac{\mathrm{d} P_{2}}{\mathrm{~d} t}=A_{21} P_{12}=\frac{P_{2}}{\tau_{\text {decay }}} .
$$

The first form in Equation (6) represents the rate of decay of an infinitely massive excited state alone in a va-

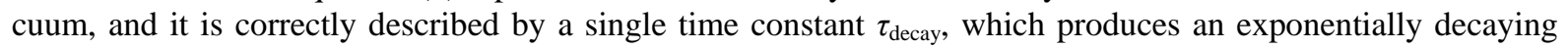

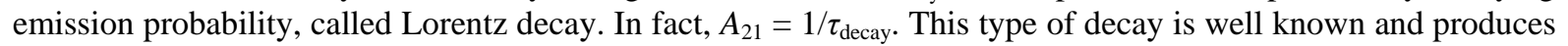
a probability distribution of frequencies called a Lorentz emission spectrum, with a frequency probability given in Equation (7a) [2], where $v_{o}=$ the center frequency of the decay spectrum, and $\Delta v_{L}=$ the half-width of the emission line. This equation is normalized so that it integrates to unity and is thus a probability distribution of the emitted frequencies.

$$
P(v)=\frac{1}{2 \pi} \frac{\Delta v_{L}}{\left(v-v_{o}\right)^{2}+\left(\frac{\Delta v_{L}}{2}\right)^{2}}
$$




$$
\text { where } \Delta v_{L}=\frac{1}{2 \pi \tau_{\text {decay }}} \text {. }
$$

Equation (7a) is the emission spectrum of an excited state into a single lower energy state, while to compute the excitation rate we need the reverse-the absorption spectrum of the lower state into the higher state. Fortunately the two are identical, a principle often used in astronomy to measure absorption lines of atoms and molecules by using their emission caused by illumination from a nearby star [3].

To compute the effective frequency bandwidth of the emission line, we simply renormalize this distribution so that the center frequency $v_{o}$ has value 1 -indicating $100 \%$ sensitivity. To accomplish this new normalization, we simply multiply Equation (7a) by $\pi \Delta v_{L} / 2$. Since Equation (7a) integrates to one, the new function will integrate to $\pi \Delta v_{L} / 2$ - the effective frequency bandwidth of the emission as given by Equation (8). We can then substitute the value of $\Delta v_{L}=1 /\left(2 \pi \tau_{\text {decay }}\right)$ from Equation $7(\mathrm{~b})$ to get $A_{21}$ in terms of the effective frequency bandwidth in Equation (9).

$$
\Delta f_{\text {eff }}=\frac{\pi}{2} \Delta v_{L}=\frac{1}{4 \tau_{\text {decay }}}
$$

Now we can write Equation 5(a) as

$$
A_{21}=B_{21} \frac{8 \pi h v^{3}}{c^{2}}=\frac{1}{\tau_{\text {decay }}}=4 \Delta f_{\text {eff }} .
$$

Solving for $B_{21}$, we get the following result with a little algebra, which must also equal $B_{12}$ since the two are equal from Equation (5b).

$$
B_{21}=B_{12}=\frac{\Delta f_{\text {eff }}\left(\frac{\lambda^{2}}{2 \pi}\right)}{h v}
$$

This result becomes very physical in Equation (11) when we compute the rate of excitation (ER) for an unexcited atom as defined in Einstein's paper above.

$$
E R=P_{1} B_{12} B B(v)=P_{1} B B(v) \frac{\Delta f_{e f f}\left(\frac{\lambda^{2}}{2 \pi}\right)}{h v}
$$

Now we rewrite this equation in terms of the $P_{F R}$ (photon flux rate per unit area per frequency), which is given simply as Equation (12).

$$
P_{F R}=\frac{B B(v)}{h v} \quad\left(\text { photons } / \mathrm{sec} / \mathrm{cm}^{2} / \mathrm{Hz}\right) .
$$

The excitation rate per second (ER) is then

$$
E R=P_{1} P_{F R} \Delta f_{e f f}\left(\frac{\lambda^{2}}{2 \pi}\right) .
$$

To identify the term we seek, the general form to compute an excitation rate is by taking the probability of being unexcited $\left(P_{1}\right)$ times the number of photons $/ \mathrm{sec} / \mathrm{cm}^{2} / \mathrm{Hz}\left(P_{F R}\right)$ times the effective bandwidth of the excitation $\left(\Delta f_{\text {eff }}\right)$ times the cross-section of interaction $\left(C_{\text {photon }}\right)$ for the photon to give photons/sec excited, as shown in Equation (14).

$$
E R=P_{1} P_{F R} \Delta f_{\text {eff }} C_{\text {photon }}
$$

We see immediately that the cross-section of the photon for every excitation of a heavy electronic system in its rest frame must be

$$
C_{\text {photon }}=\frac{\lambda^{2}}{2 \pi}
$$

where $\lambda=$ the wavelength in the rest frame of our infinitely heavy atom. 


\section{Conclusions}

The model of an infinitely heavy two-state electronic system in thermal equilibrium used by Einstein in his 1917 paper [1] leads to a universal photonic cross-section equal to $\lambda^{2} / 2 \pi$. The only additional mathematics we needed was the spectrum of Lorentz emission, a well known process both in QM and experimental physics for spontaneous decay [2]. Einstein had assumed that this form of emission was his equilibrium analysis by making the probability $P_{2}$ of the excited state decay at a rate $P_{2} / t_{\text {decay }}$. Given that the only apparent assumptions here are blackbody equilibrium and Lorentz decay combined with the three physical processes of excitation, spontaneous decay and stimulated emission, the cross-section derived would appear to be quite general.

This result is satisfying in that it makes the effective area of a photonic interaction proportional to $\lambda^{2}$. It seems likely that suitable QM calculations should come to a similar conclusion.

Such a simple result can be quite useful in physical calculations. For instance, suppose one has an atom or molecule of any type on a solid surface and needed to know what photon flux it would take to excite it well. If you irradiate it with a laser frequency tuned to its transition, then each atom or molecule will be excited at a rate equal to the photon flux/unit area times $\lambda^{2} / 2 \pi$. In this way all the details of the transition can be ignored and active sensors of trace chemicals can be easily designed.

\section{References}

[1] Einstein, A. (1917) Zur Quantentheorie der Strahlung (On the Quantum Theory of Radiation). Physika Zeitschrift, 18, 121-128.

[2] www.pci.tu-bs.de/aggericke/PC4e/Kap_III/Linienbreite.htm

[3] Sharma, A. and Schulman, S.G. (1999) Introduction to Fluorescence Spectroscopy. Wiley Interscience, Hoboken. 